\title{
The growth of HgSe by molecular beam epitaxy for ohmic contacts to $\mathrm{p}-\mathrm{ZnSe}$
}

\author{
S. Einfeldt *, H. Heinke, M. Behringer, C.R. Becker, E. Kurtz, D. Hommel, \\ G. Landwehr
}

Physikalisches Institut der Universität Würzburg, Am Hubland, D-97074 Würzburg, Germany

\begin{abstract}
The structural properties of HgSe grown by molecular beam epitaxy (MBE) are investigated for different lattice mismatches to the substrate and various growth conditions. The growth rate is shown to depend strongly on the growth temperature above $100^{\circ} \mathrm{C}$ as well as on the $\mathrm{Hg}$ /Se flux ratio. It has been found that the crystalline perfection and the electrical properties are mainly determined by the layer thickness, especially for the growth on highly lattice mismatched substrates. Changes in the surface morphology are related to growth parameters. Differences between the electrical behavior of MBE-grown and bulk $\mathrm{HgSe}$ are discussed. The electrical properties of $\mathrm{HgSe}$ contacts on $\mathrm{p}-\mathrm{ZnSe}$ are investigated as a function of different annealing procedures.
\end{abstract}

\section{Introduction}

The fabrication of low-resistance, ohmic contacts to $\mathrm{p}-\mathrm{ZnSe}$ is still one of the major problems in the technology of blue and green light emitting devices based on $\mathrm{ZnSe}$ and related compounds. At present, gold is the most commonly used contact material which forms a Schottky contact with an offset of about $1.4 \mathrm{eV}$ to the valence band of $\mathrm{ZnSe}$. To obtain light emission, large voltages of 10-20 $\mathrm{V}$ are needed to break down the reverse biased $\mathrm{Au} / \mathrm{p}-\mathrm{ZnSe}$ junction in a forward biased diode device.

Recently Lansari et al. [1] produced nearly ohmic contacts to highly doped $\mathrm{p}-\mathrm{ZnSe}$ by using $\mathrm{HgSe}$. They observed smaller LED turn-on voltages of 2-3 V compared to that of gold as a

\footnotetext{
* Corresponding author.
}

result of the lower valence band offset of $0.6-0.8$ $\mathrm{eV}$ [2]. A p-type doped $\mathrm{Hg}_{1-x} \mathrm{Zn}_{x}$ Se graded layer was predicted to further improve the diode properties [1,3].

The potential of $\mathrm{HgSe}$ as a contact to p-type $\mathrm{ZnSe}$ has lead to an increased interest in investigations of its MBE growth conditions. Until now very few publications concerning this matter have appeared in the literature $[1,4]$. In this article, characteristics of $\mathrm{MBE}$ growth of $\mathrm{HgSe}$ are presented and their influence on structural and electrical properties of the resulting epilayers are discussed.

\section{Experimental details}

Epitaxial growth was carried out in a Riber $2300 \mathrm{MBE}$ system, equipped with a self-designed $\mathrm{Hg}$ effusion cell. The absolute Se flux was rou- 
tinely calibrated by depositing Se on cold GaAs and Si substrates, assuming the Se sticking coefficient to be unity. HgSe was grown on (001) CdTe and (001) ZnTe substrates, which have a lattice mismatch of $6.1 \%$ and $0.27 \%$, respectively. The substrates were chemo-mechanically polished with a solution of $\mathrm{Br}$ in methanol, degreased in standard solvents, etched with $\mathrm{HCl}$, thermally cleaned in vacuum at $350^{\circ} \mathrm{C}$ (under $\mathrm{Cd}$ flux for the CdTe substrates) and overgrown with a homoepitaxial buffer layer. Nitrogen-doped, p-type ZnSe layers were grown on GaAs (001) substrates in a separate MBE growth chamber and transferred to the $\mathrm{Hg}$ chamber without breaking the ultrahigh vacuum (UHV).

The thickness of the $\mathrm{HgSe}$ epilayers on all samples was determined directly with a depth profiler by measuring the height of a step caused by a contact mask on the substrates. The electrical properties were determined using the Van der Pauw method for the Hall effect at $0.3 \mathrm{~T}$. The data were analyzed with a one-charge carrier model. The crystalline quality of the epilayers was investigated by means of the (004) Bragg reflection in a high-resolution five-crystal $X$-ray diffractometer. Electrochemical $C-V$ profiling was used to determine the carrier concentration in the ZnSe: $N$ layers.

\section{Results and discussion}

The growth rate of $\mathrm{HgSe}$ depends strongly on the growth temperature, as shown in Fig. 1. This indicates a much higher desorption rate for $\mathrm{HgSe}$ than for $\mathrm{HgTe}$ whose growth rate remains nearly constant over the same temperature range [5]. The exponential fit indicated in Fig. 1 gives an activation energy for the desorption of only 0.22 $\pm 0.03 \mathrm{eV}$. This value is lower than 0.51 and 0.52 $\mathrm{eV}$, which are the congruent heats of sublimation for $\mathrm{Hg}(\mathrm{g})$ and $\mathrm{Se}_{n}(\mathrm{~g})$, respectively, in equlibrium with crystalline $\mathrm{HgSe}$ [6]. Heating $\mathrm{HgSe}$ in UHV shows that chemi-desorption is negligible for temperatures below $200^{\circ} \mathrm{C}$. Therefore the observed decrease in the growth rate is related to the precursor state and not to the chemisorbed state. It should be mentioned that one is limited to

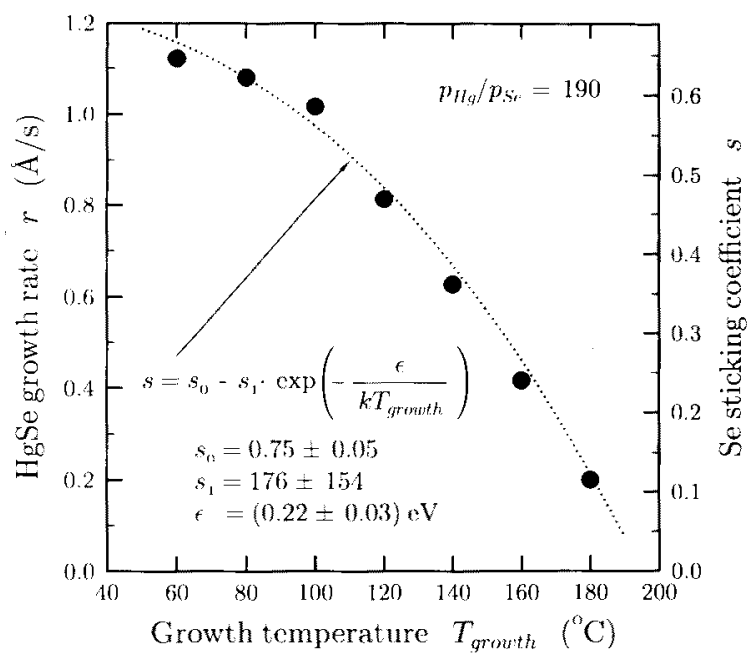

Fig. 1. The growth rate of $\mathrm{HgSe}$ and the Se sticking coefficient versus the growth temperature.

growth temperatures lower than $120^{\circ} \mathrm{C}$ for useful growth rates of $\mathrm{HgSe}$.

In addition to its dependence on the growth temperature, the growth rate is also influenced by the $\mathrm{Hg}$ flux. Fig. 2 indicates the presence of distinctly different behavior of the growth rate for two ranges of $\mathrm{Hg}$ fluxes. The growth rate increases with increasing $\mathrm{Hg} / \mathrm{Se}$ beam pressure

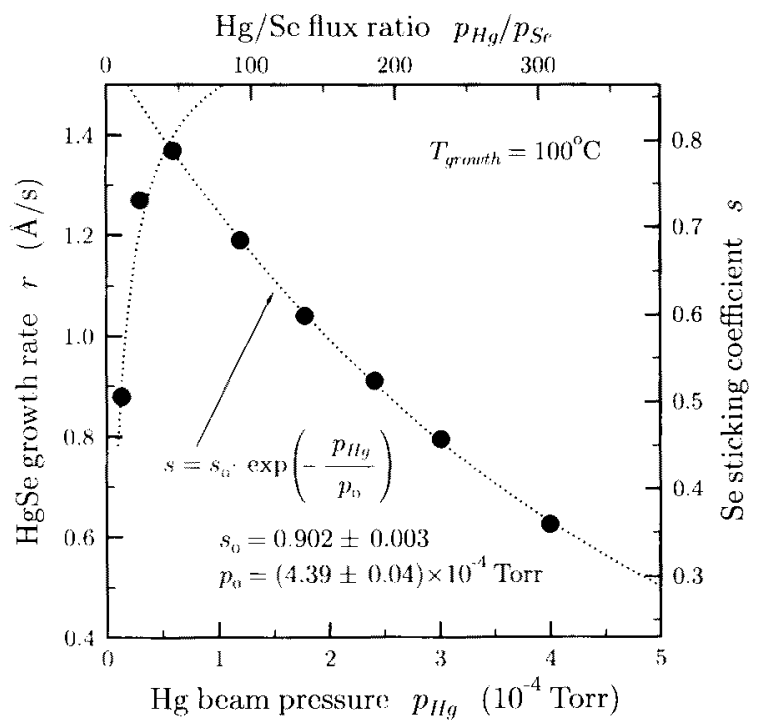

Fig. 2. The growth rate of $\mathrm{HgSe}$ and the Se sticking coefficient versus the $\mathrm{Hg}$ beam pressure and $\mathrm{Hg}$ / Se flux ratio. 
ratio (in the following loosely referred to as flux ratio) as long as this ratio is lower than 50 . This behavior is well known for the MBE growth of binary compounds $\mathrm{AB}$ (see, e.g., ref. [7]) and is indicative of growth under B-rich conditions, i.e. Se-rich conditions. This is the case in spite of the apparent excess of $\mathrm{Hg}$, due to the extremely low $\mathrm{Hg}$ sticking coefficient. Polycrystalline growth occurs for $\mathrm{Hg} / \mathrm{Se}$ flux ratios smaller than 20 . For $\mathrm{Hg} /$ Se flux ratios higher than 50 , the growth rate decreases exponentially, which might have several causes. A possible explanation is the prevention of the incorporation of Se into the crystal due to screening of the surface by a high surface concentration of $\mathrm{Hg}$ atoms in a physisorbed precursor state. Another mechanism could be the scattering of the $\mathrm{Se}_{n}(n=6,5,7, \ldots)$ molecules by the high density of $\mathrm{Hg}$ atoms in the $\mathrm{Hg}$ beam. Recently, a similar but less obvious dependence on $\mathrm{Hg}$ flux was determined for the growth of $\mathrm{HgTe}$ by means of oscillations in the reflection of high-energy electron diffraction (RHEED) [5]. It is unclear at the present as to which mechanism (or combination of both) is responsible for the large decrease in $\mathrm{HgSe}$ growth rate.

The dependence of the growth rate on both the growth temperature and the $\mathrm{Hg}$ flux led us to study the structural properties of the HgSe layers as a function of their thickness in detail. Fig. 3 demonstrates that the crystalline quality of $\mathrm{HgSe}$ grown on highly lattice-mismatched CdTe depends strongly on the layer thickness. The best HgSe samples, i.e. thick layers, have rocking curves with a full width at half maximum (FWHM) down to 220 arc sec. The large dislocation density at the $\mathrm{HgSe} / \mathrm{CdTe}$ interface becomes smaller with increasing thickness due to a healing process. The growth on nearly lattice-matched $\mathrm{ZnTe}$ provides partially strained layers which relax at a critical thickness $d_{\mathrm{c}}$ with subsequent generation of dislocations. This is clearly demonstrated by the maximum in Fig. 3 at $d=0.5 \mu \mathrm{m}$. Hence the critical thickness for $\mathrm{HgSe}$ on $\mathrm{ZnTe}$ must be smaller than $0.5 \mu \mathrm{m}$, which is in fair agreement with the value of $d_{\mathrm{c}}=0.25 \mu \mathrm{m}$ obtained from the model of Matthews and Blakeslee [8]. Other models give values as small as $23 \mathrm{~nm}$ [9] or as large as $2.4 \mu \mathrm{m} \mathrm{[10].}$

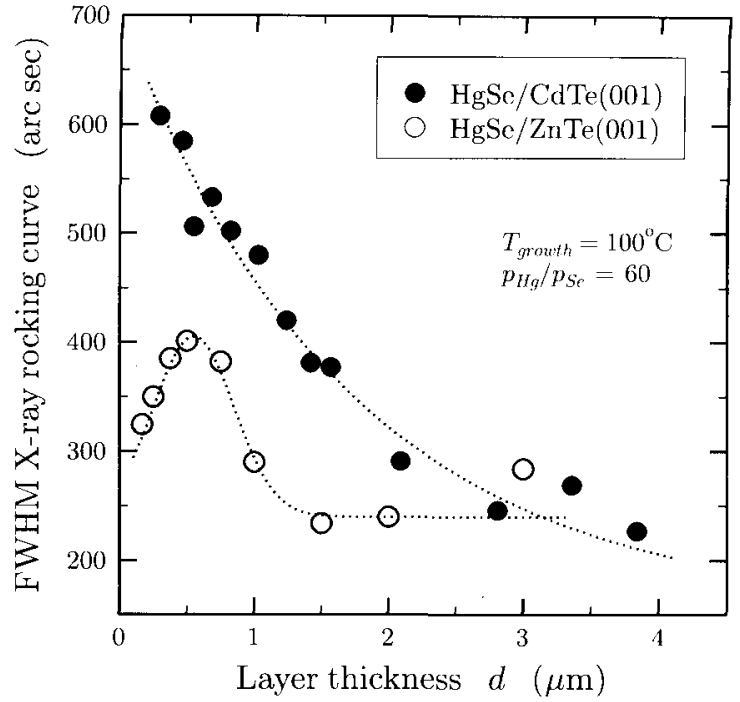

Fig. 3. Full width at half maximum (FWHM) of the (004) $\mathrm{X}$-ray rocking curves versus the layer thickness of $\mathrm{HgSe}$ grown on CdTe and ZnTe.

The change of the crystalline quality with layer thickness is accompanied by a change in the electrical properties, as shown in Fig. 4 for the growth on CdTe. The mobility at $300 \mathrm{~K}$ is limited mainly by the crystal perfection which gets worse near the substrate interface. Moreover, the Hall data indicate that the higher dislocation density causes an increase in the carrier concentration,

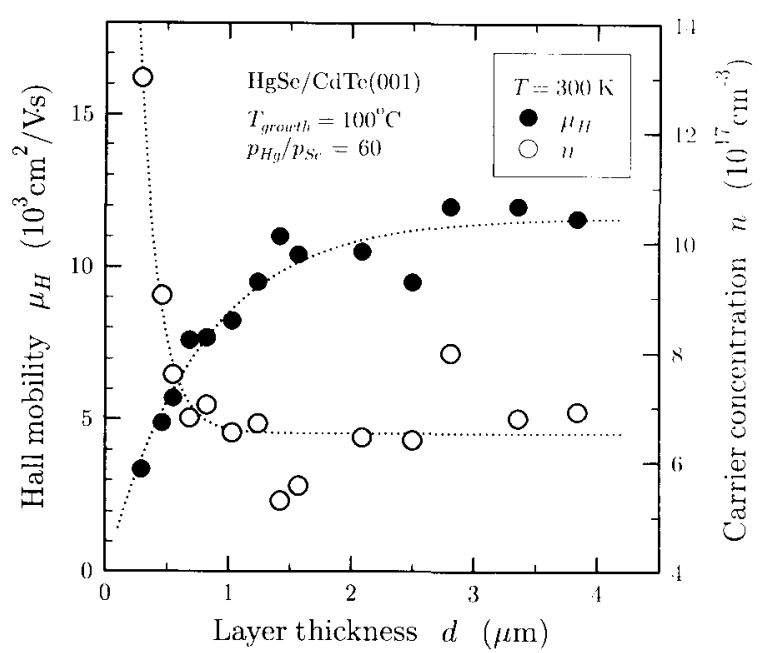

Fig. 4. Hall mobility and carrier concentration at $300 \mathrm{~K}$ versus layer thickness of $\mathrm{HgSe}$ grown on CdTe. 
i.e. is related with the generation of donors. However, this is inconsistent with measurements of the interband absorption whose interpretation requires a nearly constant carrier concentration [11]. In the case of the $\mathrm{MBE}$ growth of $\mathrm{HgSe}$ on highly lattice-mismatched CdTe substrates, the dependence of the crystalline quality on the layer thickness is shown to be much stronger than its dependence on any growth parameter, i.e. growth temperature or $\mathrm{Hg} / \mathrm{Se}$ flux ratio. Mobilities of the best, i.e. thick, epilayers range from $12000 \mathrm{~cm}^{2} / \mathrm{V}$ - s at $300 \mathrm{~K}$ to $44000 \mathrm{~cm}^{2} / \mathrm{V} \cdot \mathrm{s}$ at $20 \mathrm{~K}$.

The surface of $\mathrm{HgSe}$ samples exhibits rectangular pyramidal hillocks similar to those observed on epitaxially grown (001) $\mathrm{HgCdTe}$ (see, e.g., ref. [12]). The density of these hillocks is $6 \times 10^{4}$ to $5 \times 10^{5} \mathrm{~cm}^{-2}$. Furthermore, an additional "macroscopic" roughness was observed which depends on the growth parameters. Surface scans presented in Fig. 5 show a decrease in the roughness with decreasing growth temperature and increasing $\mathrm{Hg} / \mathrm{Se}$ flux ratio. The surface roughening is possibly related to the $\mathrm{Hg}$ coverage of the growth

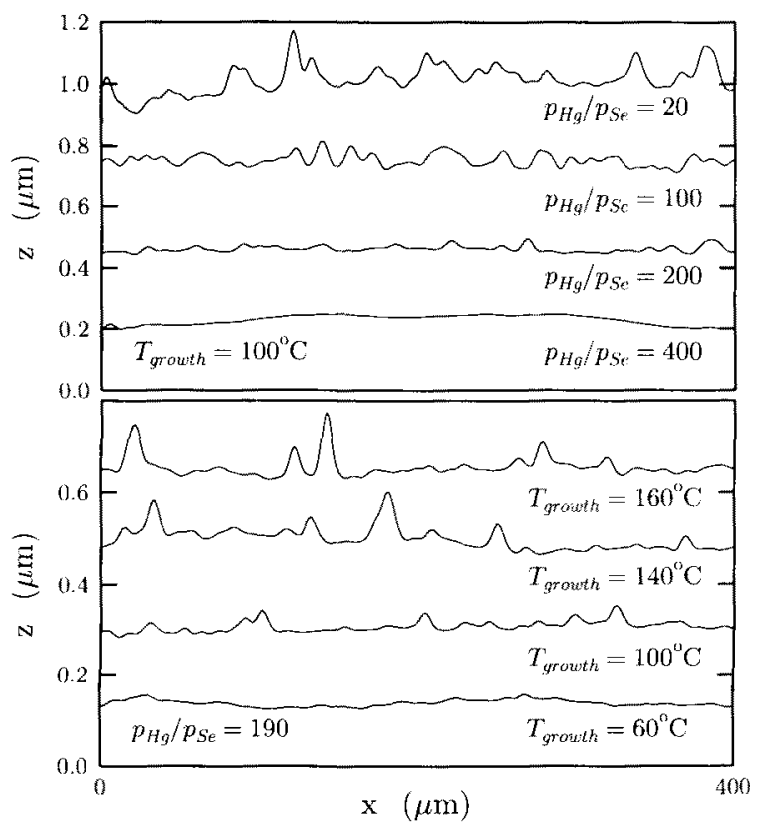

Fig. 5. Surface roughness of $\mathrm{HgSe}(d=0.6 \mu \mathrm{m})$ on CdTe for different growth temperatures and $\mathrm{Hg} / \mathrm{Se}$ flux ratios.

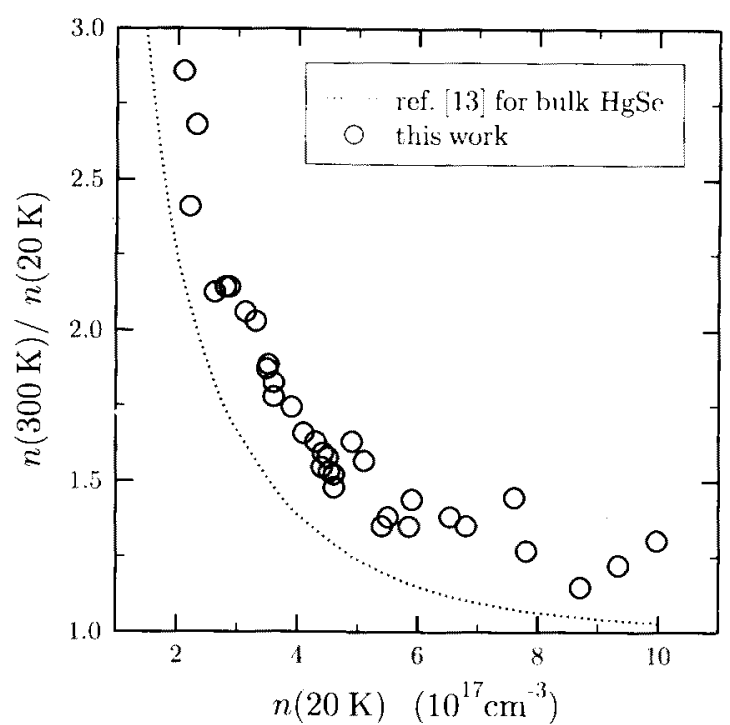

Fig. 6. Ratio of carrier concentrations at 300 and $20 \mathrm{~K}$ versus carrier concentration at $20 \mathrm{~K}$.

surface, which decreases with higher temperatures and lower $\mathrm{Hg}$ fluxes.

Comparing the electrical data of our samples to bulk $\mathrm{HgSe}$, we observe a stronger than expected decrease in the carrier concentration at lower temperatures, as shown in Fig. 6. The dashed line is the expected behavior, calculated using the method and parameters published for bulk $\mathrm{HgSe}$ [13]. Assuming that the band structure parameters are correct, the deviation for MBE grown $\mathrm{HgSe}$ can be explained by (1) postulating more than one type of charge carriers, (2) assuming a temperature dependence for the donor concentration, or (3) supposing different scattering mechanisms which would change the carrier concentration as calculated from the experimental Hall constant. At present none of these explanations could be shown to be the correct one.

In order to study the electrical properties of $\mathrm{HgSe}$ contacts on $\mathrm{p}$ - $\mathrm{ZnSe}$, contact stripes (3.1 $\mathrm{mm} \times 0.5 \mathrm{~mm})$ of $\mathrm{p}-\mathrm{ZnSe}\left(p=4 \times 10^{17} \mathrm{~cm}^{-3}\right)$ with five equidistant mesas $(0.1 \mathrm{~mm} \times 0.5 \mathrm{~mm})$ of $\mathrm{HgSe}(250 \mathrm{~nm}) / \mathrm{Ni}(30 \mathrm{~nm}) / \mathrm{Au}(220 \mathrm{~nm})$ were fabricated using standard photolithographic, etching and lift-off techniques. Three- and four-point measurements provided the contact resistance and the resistivity of the p-ZnSe layer, respec- 
tively. The samples were annealed in nitrogen atmosphere in order to produce a graded gap $(\mathrm{HgSe}-\mathrm{ZnSe})$ by $\mathrm{Hg}-\mathrm{Zn}$ interdiffusion [14]. I-V curves measured between two $\mathrm{HgSe}$ contacts after annealing for different periods of time at $300^{\circ} \mathrm{C}$ are shown in Fig. 7. By extrapolating the linear part of the curve at high voltages to $I=0$, typical voltage offsets of $1-2 \mathrm{~V}$ are obtained which are consistent with the values reported in ref. [1] for this hole concentration in $\mathrm{p}-\mathrm{ZnSe}$. Annealing the samples does not result in a ohmic behavior of the contacts, as has been shown for $\mathrm{HgTe} / \mathrm{p}-\mathrm{CdTe}$ contacts [15], but increases the total resistance as illustrated in Fig. 7. This is mainly due to an increase in the resistivity of the p-ZnSe layer during annealing, as determined by four-point measurements, see Fig. 8 . $C-V$ measurements showed that the hole concentration in $\mathrm{p}-\mathrm{ZnSe}$ decreased by annealing, but only in a 50 nm thick region near the surface by less than a factor of 4 . Since this change in the hole concentration is too small to explain the increase in the resistivity, annealing seems to reduce the hole mobility. The physical background of this effect is not understood at present.

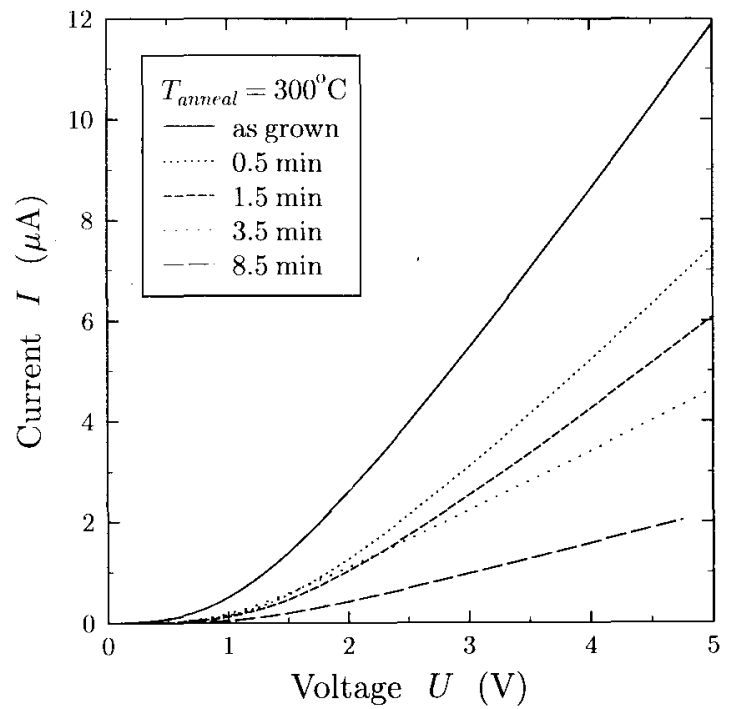

Fig. 7. Two-point measurements of current versus voltage for $\mathrm{p}-\mathrm{ZnSe}$ with $\mathrm{HgSe}$ contacts after annealing at $300^{\circ} \mathrm{C}$ for different periods of time.

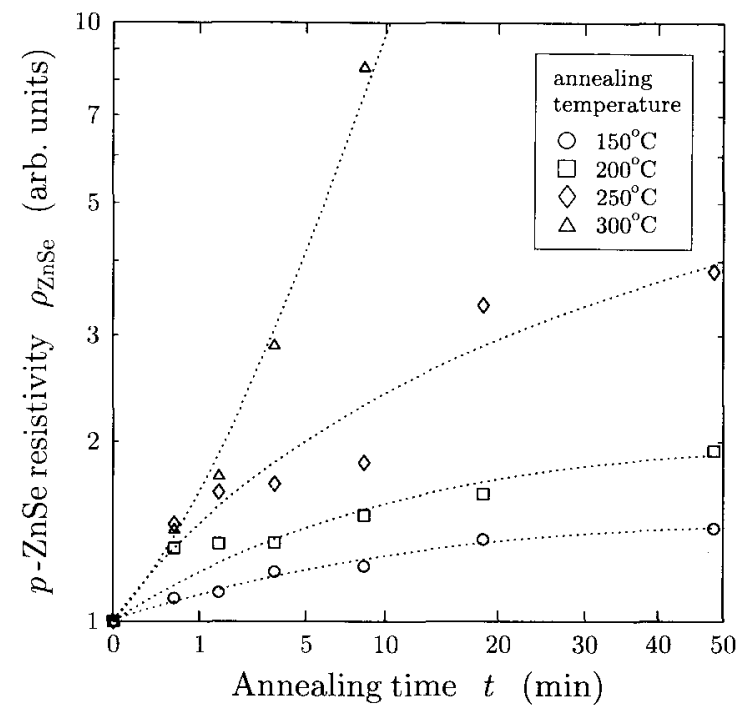

Fig. 8. Resistivity of a p-ZnSe layer determined by four-point measurements versus annealing time for different annealing temperatures.

The contact is not ohmic, as can be seen in the nonlinear $I-V$ curves of Fig. 7. However, the voltage drop across the contact as determined by three-point measurements was taken as a figure of merit for the contact. This value was reduced by 0.5 to $1.5 \mathrm{~V}$ upon annealing at temperatures up to $200^{\circ} \mathrm{C}$. All experimental results lead to the conjecture that ohmic behavior of $\mathrm{HgSe} / \mathrm{p}-\mathrm{ZnSe}$ contacts cannot be obtained by annealing and that a graded gap between $\mathrm{HgSe}$ and $\mathrm{p}-\mathrm{ZnSe}$, which should display ohmic behavior, has to be grown by MBE.

\section{Conclusions}

The influence of MBE growth parameters on the structural properties of $\mathrm{HgSe}$ was investigated. The growth rate was shown to depend on the growth temperature and the $\mathrm{Hg} / \mathrm{Se}$ flux ratio. For the growth on highly lattice-mismatched substrates, the crystal perfection is determined mainly by the layer thickness and less by growth parameters. MBE-grown $\mathrm{HgSe}$ shows significant differences in electrical behavior compared to bulk $\mathrm{HgSe}$. Electric contacts to $\mathrm{p}-\mathrm{ZnSe}$ by means 
of $\mathrm{HgSe}$ do not exhibt ohmic behavior after annealing, whereas the resitivity of annealed $\mathrm{p}-\mathrm{ZnSe}$ increases significantly.

\section{Acknowledgements}

This work was supported by the Bundesminesterium für Forschung und Technologie, Bonn, Germany. We would like to thank A. Schönteich for maintaining the MBE system, P. Wolf-Müller and T. Schuhmann for preparing the substrates and $\mathrm{M}$. Koubek for fabricating the contacts.

\section{References}

[1] Y. Lansari, J. Ren, B. Sneed, K.A. Bowers, J.W. Cook, Jr. and J.F. Schetzina, Appl. Phys. Lett. 61 (1992) 2554.

[2] J.S. Best, J.O. McCaldin, T.C. McGill, C.A. Mead and J.B. Mooney, Appl. Phys. Lett. 29 (1976) 433.
[3] Z. Yang and J.F. Schetzina, 1993 Electronic Materials Conf., Santa Barbara, CA, June 1993.

[4] C.R. Becker, L. He, S. Einfeldt, Y.S. Wu, G. Lérondel, H. Heinke, S. Oehling, R.N. Bicknell-Tassius and G. Landwehr, J. Crystal Growth 127 (1993) 331.

[5] S. Oehling, Diploma Thesis, University of Würzburg (1993).

[6] R.F. Brebrick, J. Chem. Phys. 43 (1965) 3846.

[7] Th. Litz, Th. Behr, D. Hommel, A. Waag and G. Landwehr, J. Appl. Phys. 72 (1992) 3492.

[8] J.W. Matthews and A.E. Blakeslee, J. Crystal Growth 27 (1974) 118.

[9] J.H. Van der Merwe, J. Appl. Phys. 34 (1963) 123.

[10] R. People and J.C. Bean, Appl. Phys. Lett. 47 (1985) 322.

[11] S. Einfeldt, F. Goschenhofer, C.R. Becker and G. Landwehr, to be published.

[12] A. Million, L. Di Cioccio, J.P. Gailliard and J. Piaguet, J. Vac. Sci. Technol. A 6 (1988) 2813.

[13] S.L. Lehoczky, J.G. Broerman, D.A. Nelson and C.R. Whitsett, Phys. Rev. B 9 (1974) 1598.

[14] V. Leute, H. Plate and H.M. Schmidtke, Phys. Status Solidi (a) 125 (1991) 217.

[15] E. Janik and R. Triboulet, J. Phys. D (Appl. Phys.) 16 (1983) 2333. 